\title{
Occurrence of osteoarthritis of the peripheral joints in European populations
}

\author{
Ingemar F Petersson
}

The study of the occurrence of osteoarthritis, as well as other joint disorders, can be divided into estimates of the incidence and of the prevalence. However, the studies of incidence rates in European populations are so few that most information in this overview will refer to prevalence figures

Reports of osteoarthritis of peripheral joints, that is, generalised osteoarthritis, ${ }^{1}$ had already appeared during the 19th century, but studies of the occurrence of the disease in the general population did not start on a large scale until the 1940 s. $^{2}$ Among the first studies undertaken were those by Kellgren and Lawrence ${ }^{3}$ in the United Kingdom. Although many surveys have been performed since then, the prevalence figures as well as the radiographic definitions used in those studies are still of interest.

A new aspect of the study of occurrence is the paleopathological investigations that both form a basis for theories on pathogenesis ${ }^{4}$ and make it possible to study the occurrence in certain populations in the past. Osteoarthritic lesions are the most common pathological findings in preindustrial populations. ${ }^{5-7}$

A general problem when reviewing the occurrence of radiographic osteoarthritis is differences in the criteria and definitions that have been used. Several attempts have been made to create a common basis for classification, but comparison between different studies is still complicated by diverse nomenclature. ${ }^{89}$

\section{Occurrence}

PREVALENCE

A problem with disease definition impinges on estimates of osteoarthritis prevalence. All major studies are based on radiographic diagnosis, most often graded according to the Kellgren and Lawrence classification, ${ }^{10}$ but comparisons with other grading systems have been performed for some joints, such as the knee joint. ${ }^{8}$

The prevalence varies widely between different joints. The hands, knees, and hips are the most frequent locations in peripheral joints. ${ }^{11}$ Another major determinant of the occurrence is age. ${ }^{12}$ The condition hardly exists below the age of 30 but is present in the finger joints in more than $90 \%$ of people over the age of 79 years. ${ }^{13}$ The prevalence rate increases in the hands and knees up to the age of 75 but not thereafter. ${ }^{13}$ Sex and race also influence the figures, and all these factors have to be taken into account when evaluating prevalence rates.

A basis for a more "general" estimate of the presence of osteoarthritis in the general population can be made from a Swedish survey where the overall prevalence of "clinically significant" osteoarthritis in hands, hips, and knees was $5.8 \%$ in the age range $50-70$ years. ${ }^{14}$

Pain and other symptoms from the affected joints are important determinants of disability. Figures for pain in osteoarthritis populations vary between $40 \%$ and $80 \%,{ }^{15}$ but in many studies the presence and severity of symptoms have not been recorded.

In this paper the focus will be on the occurrence of radiographic osteoarthritis in the small joints of the hand, the hip joints, and the knee joints.

\section{Occurrence in different joints}

SMALL JOINTS OF THE HAND

In osteoarthritis of the hand, as well as in other joints, precise definition of the condition under study is crucial. ${ }^{16-18}$ The best method for defining osteoarthritis of the hand for epidemiological studies is radiography, but if this is not available, clinical examination may be used. ${ }^{19}$

Osteoarthritis of the finger joints is already present radiographically by 40-49 years in about $10 \%$ of individuals (slight radiographic osteoarthritis). ${ }^{12}$ The prevalence increases rapidly with age and by $70+$, more than $90 \%$ of female population and $80 \%$ of the male population are affected. ${ }^{12} 20$ Including all individuals over the age of 15 years gives prevalence figures of $22 \% / 29 \%$ (males/females) for the DIP joints. Only $9 \% / 26 \%$ (males/females) of those with Kellgren and Lawrence grade 3-4 had pain. ${ }^{2}$

In a comparison between two populations aged 70-79 years using similar methodology, $92 \%$ in Göteborg and $75 \%$ in Zoetermeer, Holland, had radiographic hand osteoarthritis. ${ }^{20}$

HIP JOINTS

Prevalence rates of osteoarthritis of the hip joint at 55 years and above are much lower than for the knee and finger joints. In a study by Kellgren and Lawrence the prevalence of hip osteoarthritis was $8.4 \%$ in men and $3.1 \%$ in women (Kellgren and Lawrence grade 3 or 4 ). ${ }^{3}$ 
In a survey in Denmark, Jørring found a prevalence of $5.6 \%$ in women and $3.7 \%$ in men over 60 years of age, with bilateral disease in $44 \%$. $^{21}$

In a Swedish survey of colonic $x$ rays (over the age of 55 years) the prevalence "primary coxarthrosis" was $3.4 \%$ (no sex difference, bilateral in $31 \%$ of the cases). ${ }^{22}$ Twenty years later Lindberg found a total prevalence of hip osteoarthritis of $2.1 \%$ and $3.1 \%$ over the age of 55 years (no sex difference, 35\% bilateral disease) in a Swedish survey of normal colon radiographs. ${ }^{23}$ Thus a comparison of prevalence figures between the two identically performed studies showed no changes over a 20 year time period.

The Zootermer survey in Holland showed in the age group 45-49 years that the prevalence of hip osteoarthritis was approximately 3\% (Kellgren and Lawrence grade 2 or more) ${ }^{24}$ In the US National Health Survey Lawrence et al found prevalence rates of hip osteoarthritis (Kellgren and Lawrence grades $2-4$ ) of $2.6 / 2.8 \%$ (males/females) in the age group 55-64 years and $4.6 / 2.7 \%$ in the age group $65-74 .^{25}$

KNEE JOINTS

Depending on the populations studied and the epidemiological techniques used, the prevalence figures for radiographic osteoarthritis of the knees (with or without symptoms) range from $3.8 \%$ (ages 25-74 years) ${ }^{25}$ to between $14 \%$ and $30 \%$ (over the age of 45 years). ${ }^{15}$

A diagnosis of osteoarthritis has often been based on radiographic criteria alone. Plain radiographs with different grading systems for the tibiofemoral compartment of the knee have been used, for example, the Kellgren and Lawrence system $^{10}$ and the Ahlbäck classification. ${ }^{26}$

Recent data suggest that the patellofemoral joint is also important when studying knee osteoarthritis ${ }^{27}$ but most studies so far have only dealt with the tibiofemoral joint.

In a study of radiographic knee osteoarthritis Kellgren and Lawrence found a prevalence of $40.7 \%$ in females and $29.8 \%$ in males aged 55-64 years. ${ }^{3}$ In a more recent Dutch study of subjects aged 45-49 years, the prevalence of radiographic knee osteoarthritis was between $7.7 \%$ and $14.3 \%$ (Kellgren and Lawrence grade 2 or more), depending on sex and whether it was located in the right or left knee. ${ }^{24}$ The proportion with symptoms was not mentioned in these studies. The proportion of patients with radiographic knee osteoarthritis who also have knee pain varies between $40 \%$ and $80 \%$ in most studies, but pain occurs most often in subjects aged 50 years or above. ${ }^{8}$

In the Chingford study, Spector and coworkers found symptomatic radiographic knee osteoarthritis in $2.9 \%$ of women aged 45-65 years. ${ }^{28}$

With increasing age, the osteoarthritis prevalence increases considerably and in older age groups (75-79 years) the prevalence of knee osteoarthritis is known to be high, at between $40 \%$ and $60 \% .^{13}$

In a study of a cohort of middle aged individuals (aged 35-54 years) with chronic knee pain, it was possible to calculate a minimum prevalence figure of $3.3 \%$ for radiographic knee osteoarthritis (joint space narrowing, Ahlbäck grade I or more, corresponding to Kellgren and Lawrence grade 3 or more (Peterson F, unpublished data)).

Only a few studies have addressed the question of symptomatic radiographic knee osteoarthritis. In an analysis of data from NHES and NHANES I, Lawrence and coworkers found that the prevalence of "mild + moderate + severe osteoarthritis" with and without symptoms in the age groups 35-44 and 45-54 years was $1.6 \%$ and $3.0 \%$ respectively. ${ }^{25}$ In the same age groups the prevalence of "moderate + severe osteoarthritis" (which most probably correspond to the Ahlbäck classification of grade I or more, including only cases with joint space reduction) was $0.3 \%$ and $0.4 \%$, respectively.

\section{Incidence}

As with other chronic and disabling diseases it is of interest to know the incidence figures for osteoarthritis of different joints. Because of problems of disease definition and strategies to detect new cases, very few studies of incidence had been performed until recently. ${ }^{29}{ }^{30}$ By using a strategy to ascertain new clinical attenders with radiographic osteoarthritis, it was found in a study of a US population that nearly $1 \%$ per year of women aged 70-89 years developed symptomatic knee osteoarthritis. The incidence figures were less than half that for hand osteoarthritis and hip osteoarthritis. ${ }^{29}$ The incidence increased with age. In another survey of knee osteoarthritis in elderly persons, women developed symptomatic radiographic osteoarthritis at a rate of $1 \%$ per year, and $4 \%$ per year experienced progression of existing knee osteoarthritis. Incidence rates were significantly higher in women than in men. ${ }^{30} \mathrm{In}$ European populations the Zoetermer survey included a study of the incidence of radiographic knee osteoarthritis in 258 individuals from the general population aged more than 45 years by comparing the results of two cross sectional studies. During a 12 year follow up, about $25 \%$ of the women and about $10 \%$ of the men developed radiographic knee osteoarthritis ${ }^{31}$ : over a 5 year period (between the ages of 75 and 79) the incidence of DIP and PIP osteoarthritis was $13.6 \%$, and of knee osteoarthritis $4.5 \%$. $^{13}$. In subpopulations studies have been performed on incidence rates related to specific risk factors. ${ }^{32}$

\section{Conclusion}

The data presented show that radiographic osteoarthritis of the hands, knee, and hip joints is very frequent in European populations, especially over the age of 50 years.

Many problems concerning the occurrence of osteoarthritis remain to be solved. Some of the most important tasks in this aspect are to define and determine the occurrence of "clinically important" osteoarthritis, taking into account the degree of pain, disability, and impairment of quality of life. 
Another important task for the future is to try to find methods for early diagnosis of osteoarthritis to create a basis for earlier intervention and treatment. This will include further evaluation of new diagnostic techniques, both in selected patient groups and in the general population. Such studies might in the future give us "new" and more clinically relevant prevalence figures. The question of secular trends also remains to be solved.

Much knowledge has already been gained but much more work needs to be done.

1 Lawrence JS. Generalized osteoarthritis in a population sample. Am f Epidemiol 1969;90:381-9.

2 Lawrence JS, Bremner JM, Bier F. Osteo-arthrosis. Prevalence in the population and relationship between symptoms and x-ray changes. Ann Rheum Dis 1966;25:1-24.

3 Kellgren JH, Lawrence JS. Osteo-arthrosis and disk degeneration in an urban population. Ann Rheum Dis 1958; 17:388-97.

4 Lim KKT, Rogers J, Sheepstone L, Dieppe PA. The evolutionary origins of osteoarthritis: a comparative skeletal study of hand disease in 2 primates. $f$ Rheumatol 1995;22:2132-4.

5 Rogers J, Watt I, Dieppe P. Arthritis in Saxon and medieval skeletons. BMF 1981;283:1668-70.

6 Jurmain RD, Kilgore L. Skeletal evidence of osteoarthritis: a paleopathological perspective. Ann Rheum Dis 1995; 54:443-50.

7 Rogers J, Dieppe PA. Is tibiofemoral osteoarthritis in the knee joint a new disease? Ann Rheum Dis 1994;53:612-3.

8 Spector TD, Hart DJ, Byrne J, Harris PA, Dacre JE, Doyle DV. Definition of osteoarthritis of the knee for epidemiological studies. Ann Rheum Dis 1993;52:790-4.

9 Spector TD, Hochberg MC. Methodological problems in the epidemiological study of osteoarthritis. Ann Rheum Dis 1994;53:143-6.

10 Kellgren JH, Jeffrey M, Ball J. Atlas of standard radiographs. The epidemiology of chronic rheumatism, vol 2 . Oxford: The epidemiology

11 Bagge E, Edén S, Bjelle A, Svanborg A. Osteoarthritis in the elderly. Clinical and radiographic findings in 79 and 85-years-old. Ann Rheum Dis 1991;50:535-9.

12 Kellgren JH. Osteoarthrosis in patients and populations. BMF 1961;ii: 1-6.

13 Bagge E, Bjelle A, Svanborg. Radiographic osteoarthritis in the elderly. A cohort comparison and a longitudinal study of the "70-year old people in Göteborg". Clin Rheumatol 1992:11:486-91.

14 Jacobsson L, Lindgärde F, Manthorpe R. The commonest rheumatic complaints of over six weeks duration during a rheumatic complaints of over six weeks duration during a 12-month period in a defined Swedish population: preva-
lences and relationships. Scand $\mathcal{F}$ Rheumatol 1989;18:353-60.
15 Spector T, Hart D. How serious is knee osteoarthritis? Ann Rheum Dis 1992;51:1105-6.

16 Lequesne M, Altman R. Osteoarthritis of the hand. New methods. New data. Rev Rhum 1995;62(suppl 1):1-2S.

17 Kallman DA, Wigley FM, Scott WWJ, Hochberg MC, Tobin JD. New radiographic grading scales for osteoarthritis of the hand: reliability for determining prevalence and progression. Arthritis Rheum 1989;32:1584-91.

18 Hart DJ, Spector TD, Egger P, Coggon D, Cooper C. Defining osteoarthritis of the hand for epidemiologic studies. The Chingford sudy. Ann Rheum Dis 1994;53:220-3.

19 Cicuttini FM, Spector TD. The epidemiology of osteoarthritis of the hand. Rev Rhum 1995;62(suppl1):3-8S

20 Bagge E, Bjelle A, Valkenburg H, Svanborg A. Prevalence of radiographic osteoarthritis in two elderly European populations. Rheumatol Int 1992;12:33-8.

21 Jørring K. Osteoarthritis of the hip. Epidemiology and clinical role. Acta Orthop Scand 1980;51:523-30.

22 Danielsson LG. Incidence of osteoarthrits of the hip. Clin Orthop 1966;45:67-72.

23 Lindberg H. Epidemiological studies on primary coxarthrosis. Malmö: University of Lund, 1985.

24 van Saase JLCM, van Romunde LKJ, Cats A, Vandenbroucke JP, Valkenburg HA. Epidemiology of osteoarthritis: Zoetermer survey. Comparison of radiological osteoarthritis in a Dutch population with that in 10 other populations. Ann Rheum Dis 1989;48:271-80.

25 Lawrence RC, Hochberg MC, Kelsey JL, McDuffie FC, Medsger TA, Felts WR, et al. Estimates of the prevalence of selected arthritic and muskuloskeletal diseases in the United States. F Rheumatol 1989;16:427-41.

26 Ahlbäck S. Osteoarthrosis of the knee: a radiographic investigation. Acta Radiol (Stockholm) 1968;suppl 277:772.

27 McAlindon TE, Snow S, Cooper C, Dieppe PA. Radiographic patterns of osteoarthritis of the knee joint in the community;the importance of the patellofemoral joint. Ann Rheum Dis 1992;51:844-9.

28 Spector TD, Hart DJ, Leedham-Green M. The prevalence of knee and hand osteoarthritis (OA) in the general population using different clinical criteria: the Chingford study. Arthritis Rheum 1991;34:S171.

29 Oliveria SA, Felson DT, Reed JI, Cirillo PA, Walker AM. Incidence of symptomatic hand, hip, and knee osteoarthritis among patients in a health maintenance organization. Arthritis Rheum 1995;38:1134-41.

30 Felson DT, Zhang Y, Hannan MT, Naimark A, Weissman BN, Aliabadi $\mathrm{P}$, et al. The incidence and natural history of knee osteoarthritis in the elderly. Arthritis Rheum 1995; 38:1500-5.

31 Schouten JSAG. A twelve year follow-up study on osteoarthritis of the knee in the general population. Rotterdam: Erasmus University, 1990.

32 Spector TD, Hart DJ, Doyle DV. Incidence and progression of osteoarthritis in women with unilateral knee disease in the general population: the effect of obesity. Ann Rheum Dis 1994;53:565-8.

\title{
The occurrence of osteoarthritis outside Europe
}

\author{
Peter Croft
}

A traditional epidemiological tool is to describe the distribution of a disease by personal characteristics (such as age or gender), time, and place. The argument for looking at geographical variation is that if people living in different places have different rates of disease, then it is possible to define what it is about the places which might protect against or cause the condition. In this paper I consider some of the evidence about the occurrence of osteoarthritis outside European and Caucasian populations and highlight some questions raised by this evidence. Although all osteoarthritis can be lumped together for some purposes, it is more useful to consider disease of each joint group as a separate entity. This paper will focus initially on the geographical variation of osteoarthritis in one joint in particular (the hip), but will turn to disease in other joints also.
Prevalence studies around the world have used four methods of case ascertainment: (1) radiographic surveys of the general population; (2) radiographic surveys of hospital attenders; (3) surveys of osteoarthritis patients admitted to hospital; (4) necropsy studies.

\section{General population studies}

General population surveys have to start with ancestor worship. The extraordinary studies of Kellgren and Lawrence and their international collaborators in the $1950 \mathrm{~s}$ tackled issues of observer repeatability and the need for standardised methods of disease definition in international comparisons of musculoskeletal disease long in advance of most other clinical disciplines. ${ }^{1}$

Their basic findings still stand: (1) osteoarthritis occurs everywhere, and (2) oste-
Drive, Hartshill,

Stoke-on-Trent ST4

7QB, United Kingdom

P Croft
School of Postgraduate

Medicine, Industrial

and Community

Health Research 